\title{
Improving the Energy Balance of Field-based Routing in Wireless Sensor Networks
}

\author{
Goce Trajcevski, Oliviu Ghica, Peter Scheuermann \\ Department of EECS \\ Northwestern University, Evanston, IL \\ \{gocce, oliviughica, peters\}@eecs.northwestern.edu
}

\author{
Marco Zúñiga, René Schubotz, Manfred Hauswirth \\ Digital Enterprise Research Institute \\ National University of Ireland, Galway \\ \{marco.zuniga, rene.schubotz, manfred.hauswirth\}@deri.org
}

\begin{abstract}
For high-density networks, several studies have proposed field-based routing paradigms to uniformly distribute the traffic load throughout the network. However, as network density decreases, we observe major shortcomings of the current state-of-the-art: (i) fewer number of neighbors reduce the number of available paths and leads to path merging and (ii) the paths directed towards the border of the network merge into a single path. These path merging effects decrease significantly the energy balance, and as consequence, the lifetime of the network. In this paper, we propose a novel mechanism to enable a better load balancing for single-source and multiple-source scenarios. Our evaluations demonstrate that by using the proposed methodology, the network lifetime can be prolonged between $30 \%$ and $40 \%$.
\end{abstract}

\section{INTRODUCTION}

It is a well-known fact that in Wireless Sensor Networks (WSN) settings the traditional single-shortest path algorithms tend to cause an uneven depletion of the energy resources of the network [9]. In order to overcome this limitation, multipath [6], [11], [20] has been proposed as an attractive routing paradigm for load balancing.

Several important multi-path contributions have been proposed for single-sink single-source scenarios [3], [12], [15], [18]; unfortunately, these solutions are not ideal in multiplesource deployments. When multiple sources transmit information simultaneously, together with energy balancing, it is important to reduce the number of MAC collisions occurring due to the intersection of the various paths created by the different sources. In this context, the research community has identified field-based routing [8], [14] as an efficient energy balancing mechanism for both single and multiple source scenarios. Figure 1(a) depicts an example of current fieldbased routing algorithms. The sink is positioned in the middle of the network, and 3 sources are located at the corners.

Field based routing is an important routing paradigm but it has some limitations. First, the paths that are directed towards (or close) to the edge of the network merge into a single path, which reduces significantly the energy resources of nodes at the border. Second, the number of paths selected by a source is limited to the degree of the source (number of neighbors). These effects mean that fewer nodes will have the opportunity to participate in sharing the transmission costs. Figure 1(a) depicts the limitations of the state-of-the-art. For the upper-left source (blue paths pointed by upper-left arrow), we observe that 4 different paths directed towards the edge merge into

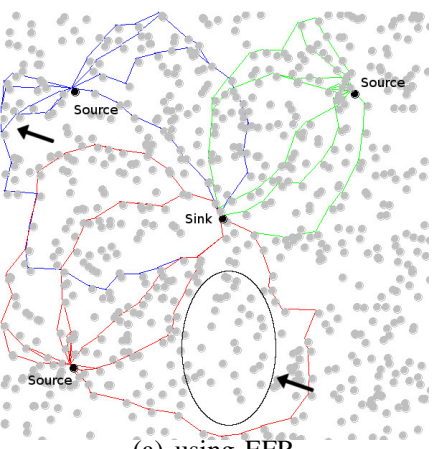

(a) using EFR

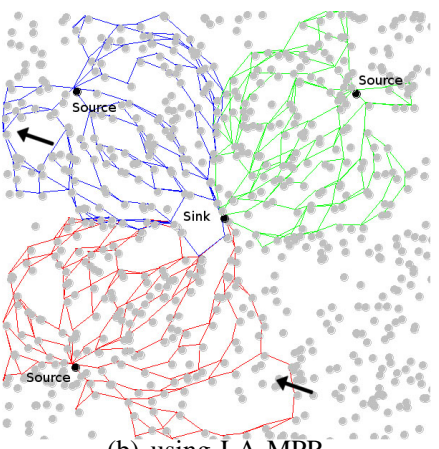

(b) using LA-MPR
Fig. 1. Comparison of the state-of-the-art EFR and our proposal LA-MPR. (a) EFR (i) fails to utilize a significant fraction of the network (bottom ellipse) and (ii) has undesirable path merging effects at the boundaries of the network (upper-left arrow). (b) LA-MPR corrects these 2 shortcomings.

one path. Also, all the sources have path merging effects that limits the balancing of energy-consumption (for instance, nodes shown by bottom-ellipse).

The main contributions of our work are two novel mechanisms to solve the path-merging limitations of field-based routing: (a) field persistent routing and (b) method of images. Figure 1(b) shows the same scenario as in Figure 1(a) but utilizing our proposed approach. We observe that our approach utilizes a larger percentage of the nodes in the network, and hence, achieves a better load balancing and prolongs network lifetime. Our results show that the energy variance in sparser networks can be reduced between $50 \%$ and $70 \%$ and the network lifetime can be improved between $30 \%$ and $40 \%$.

The next sections are organized in the following way. Section II provides some background information on fieldbased routing. The main contribution of our work is presented in Section III. The evaluation of our work is presented in Section IV, and the related literature is provided in Section V. Finally, Section VI presents our concluding remarks.

\section{PRELIMINARIES}

\section{A. Electric-Field Based Routing (EFR)}

EFR adopts the ideas of electric fields to create multiple paths. In electrostatics, the electric field $\mathbf{E}$ is represented as a set of curves that dictate the magnitude and direction of the field at any given point. In EFR, a set of these non-intersecting 


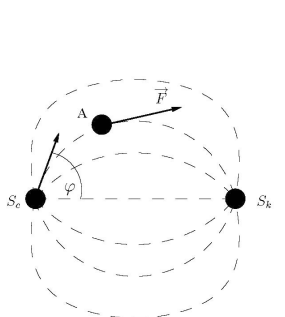

(a) Selecting a path (b) Merging of routing paths
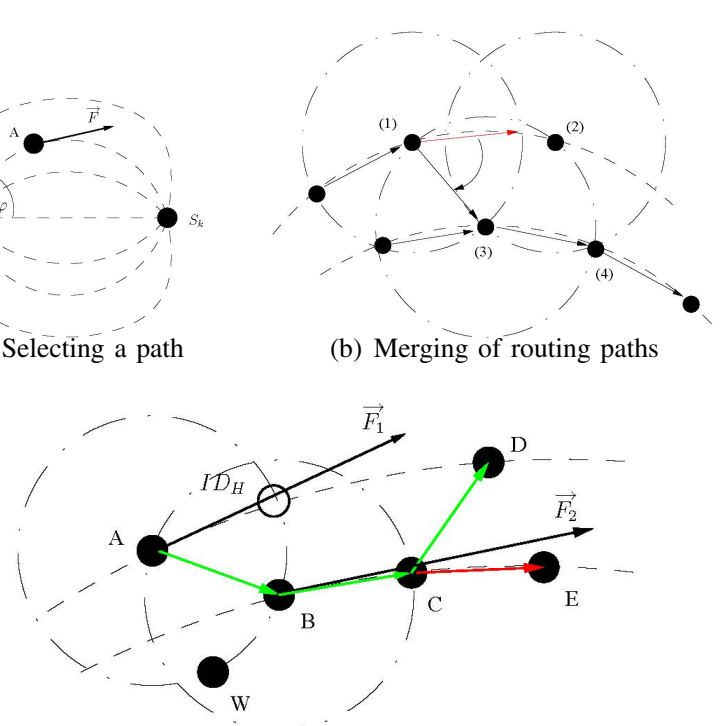

(c) Field-persistent forwarding

Fig. 2. (a) Initially the source selects an angle $\varphi$ to route the packet. This angle determines the path to be followed. (b) Depicts the path merging problem in sparse networks. (c) LA-MPR (green arrow) "unmerges" the initially merged path of EFR (red arrow), which leads to better load balancing.

electric field curves is selected as potential paths. Figure 2(a) shows a simple visualization of this process.

In our work we are interested in determining the electric field (direction of next hop) of any node $n$ located at $\mathbf{r} \in \mathbb{R}^{2}$. The electric field equation is well-known and it is given by:

$$
\mathbf{E}(\mathbf{r})=\frac{1}{4 \pi \epsilon_{0}} \sum_{i=1}^{N} \operatorname{sgn}(i) \frac{\left|q_{i}\right|}{\left|\mathbf{r}_{i}-\mathbf{r}\right|^{3}}\left(\mathbf{r}_{i}-\mathbf{r}\right)
$$

where $\mathbf{r}_{i}$ are the positions of the sink and source nodes and $\epsilon_{0}$ is the vacuum permittivity. The sink is given a negative virtual charge $\operatorname{sgn}()=$.-1 and the sources are given a positive virtual charge $\operatorname{sgn}()=$.1 . The examples presented in Figures 1(a) and 1(b) utilize this equation to form the routes.

\section{B. Network Model}

Sensor nodes are randomly deployed over the area of interest. Nodes have the capability of determining their locations, either by means of a location hardware, such as a GPS device, or by implementing a location discovery algorithm [13]. Each node has information about the locations of its onehop neighbors. We assume that nodes know the boundaries of the network (either by pre-loading this information, or by using an appropriate protocol, e.g. [5]).

\section{Limitations of Current Field-based Routing}

In EFR, at each hop, packet forwarding is based on the following rule: select the farthest 1-hop neighbor -towards the sink- that is closest to the electric field line of the current relay node. This strategy is adequate in high density networks (because of the high likelihood of having a neighbor that satisfies the required condition), but as the network density decreases, undesirable path merging effects start to appear.
We illustrate the path merging problem with Figure 2(b). According to the gradient at node (1), the next-hop is supposed to be node (2). However, since node (2) is out of range of (1), node (3) is selected. After that, node (4) is selected as next-hop of (3). This phenomena has a detrimental consequence on load balancing: while node (3) and its successors are overloaded, node (2) and its successors remain unutilized.

The second important limitation of field-based routing is the finite geographic region of WSN. EFR generates paths in all directions, as observed in Figure 1(a). When sources are close to the network's border, paths directed towards the border could merge. Some studies have proposed solutions to this problem [8], [17], but these methods yield complicated solutions, or even elude closed-form solutions.

\section{LOAD-BALANCING WITH MULTIPATH ROUTING}

We now proceed with explaining in greater detail two of the methodologies used in our routing algorithm. The detailed behavioral protocol of the sink, source(s) and relay nodes have been described in formal algorithms (available in [19]) but are not presented here due to space constraints

\section{A. Field-Persistent Routing}

Figure 2(c) illustrates the basic difference between our methodology, which we call Iso Persistent Field Routing (IFPR), and the original EFR [14]. Consider the sensor node $A$, and assume that currently it needs to forward a packet towards the sink. Node $A$ belongs to the iso-contour $\left\|\bar{F}_{1}\right\|$, however, since there are no neighbors close to this iso-contour, node $A$ switches to node $B$ in iso-contour $\left\|\bar{F}_{2}\right\|$. Then node $B$ forwards the packet to node $C$. Up to this point EFR and IFPR would behave in the same way. However, while EFR would continue the data transfer along iso-contour $\left\|\bar{F}_{2}\right\|$ (red arrow towards node $E$ ), IFPR will go back to its original iso-contour $\left\|\bar{F}_{1}\right\|$ (green arrow towards node $D$ ).

The forwarding mechanism of both EFR and IFPR is dictated by Equation 1. At each step along a route, EFR requires 3 pieces of information to select the next hop: (i) the location and charge of the source(s), (ii) the location and charge of the sink, and (iii) its own location and the location of its neighbors. On top of this, our proposal takes advantage of a simple-yet-powerful property of electric fields: the vector field applied at any point is uniquely determined by the initial angle selected at the source. Hence, by adding one more simple piece of information (the initial angle $\varphi$ ), IFPR is enabled to overcome the path-merging effects described in Section II-C. Our experiments demonstrate that the overhead of adding the angle-information $\varphi$ is negligible compared to the load-balancing benefits achieved by IFPR.

The behavior of IFPR can be intuitively explained as follows: EFR would have "forgotten" that node $D$ is the closest node to the original iso-contour, while IFPR would try to go back to the original iso-contour.

\section{B. Method of Images}

The boundary problem of EFR has been solved in [8], [17] through the use of partial differential equations. In this section, 


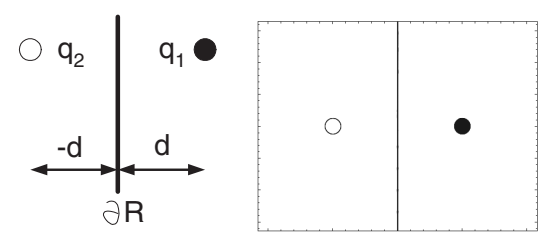

(a) at a straight-line (b) and its effect to a field boundary

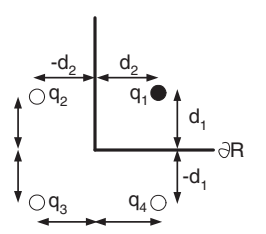

(c) at a corner of the sensing area

Fig. 3. Method of Images. (a) A virtual charge $q_{2}$ creates a zero potential at $\partial R$ (boundary of network). (b) Depicts how a virtual charge guides, in a gentle way, paths going towards the edge. (c) Shows that 3 virtual charges are used when a node is close to a corner, as opposed, to the single virtual charge that it is used when the boundary is limited by only one side.

we use a well known physical heuristic (method of images) to achieve a similar result in a simpler and distributed way.

Let us denote $\partial R$ as the boundary of the region. Our goal is to "bend-over", in a gentle way, the paths sent towards the edge in order to avoid path merging. Suppose that charge $q_{1}$ is held at a distance $d$ from the boundary, as shown in Figure 3(a). The method of images suggests to place an additional virtual charge $q_{2}$ at position $-d$ from the boundary. Considering that nodes have the geographical limits of the network (by either pre-loading it or executing an appropriate algorithm [5]), a relay node decides locally whether to apply the method of images or not. If a node observes that it is close to the border, it includes the virtual image charges in the calculation of its field vector. In Figure 3(c), we illustrate the method of images at a corner of a rectangular sensing area. As we observe, 3 image charges (white circles) are utilized in this case. If the node is close to the border but it is not close to a corner, only one image charge is utilized.

\section{Load-Aware Multipole Routing Protocol}

In Section I we stated that one of the key advantages of multi-pole electrostatic routing is its capability to reduce MAC collisions by forming mutually exclusive routing areas for each source (as shown by the colored paths in Figures 1(a) and 1(b)). In order to accomplish these mutually exclusive areas, each source needs to have up-to-date information about the existing charges (sources) in the network. Kalantari et al. [8] suggested that sink and source(s) advertise their information through flooding. Considering the drawbacks of floodingbased dissemination, in the next paragraphs we describe loadaware multi-pole routing protocol (LA-MPR), a light-weight, non-flooding protocol for the dissemination of the existing "charges" (sources) in the network.

Source Node Joining Upon query injection at the sink, the sink node assigns an electric charge $q$ to the specified source node $^{1}$. The sink keeps track of all active sources (and their charges), and communicates (i) the query and (ii) the "charges" of active sources via a $Q U E R Y$ message to the joining source node. Upon reception of a $Q U E R Y$ message, the joining

\footnotetext{
${ }^{1}$ In analogy to Coulomb's Law, the electric charge assigned to a source is reciprocally proportional to the square of the relative distance between sink and source. The charge of the sink is the negated sum of all source charges.
}

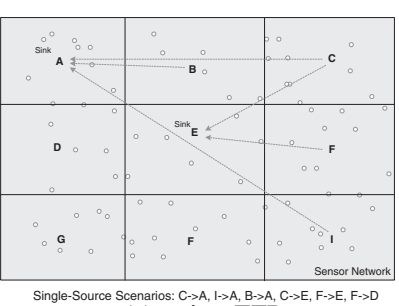

(a) using EFR

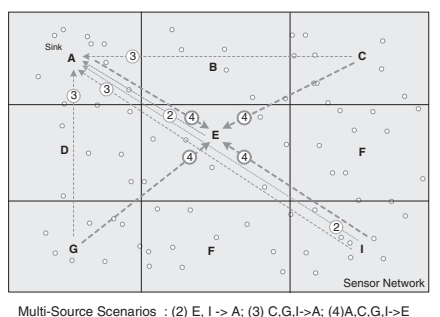

(b) using LA-MPR
Fig. 4. Deployments utilized for (a) single source and (b) multiple source scenarios. 6 different placements were used for single source. For multisource, 3 placements -with 2, 3 and 4 sources- were used. Notice that due to symmetry, these placements capture the most representative deployments.

source node resets its state, parses the user query, and starts the route discovery process.

Route Discovery The source selects outgoing angles for the required number of paths on the QUERY message, and sends $R R E Q$ messages. The paths are set to pending until they get acknowledged by the sink node via an $A C K$ message. Upon reception of a $R R E Q$ message, the sink replies to the respective source node by sending back an $A C K$ message.

Data Transmission As soon as a source has successfully discovered valid routing paths, it starts to send DATA messages using those routes. Packets are sent proportionally to the strength of the field, as suggested in [8]. Upon reception of a $D A T A$ message, the sink node extracts the message payload and returns it to the user application.

Updating The sink node keeps all active sources informed about joining sources via UPDATE messages. Upon reception of an UPDATE message, an active source updates its knowledge about other source nodes, sets its active routes to pending, and re-runs the route discovery process. This mechanism adjust the electric fields of the network according to the new source (charge). On reception of an $A C K$ messages the respective pending routes get re-activated.

Lastly, we note that the sink node transmits $A C K$, $Q U E R Y$ and $U P D A T E$ messages to the respective source nodes using a greedy geographic routing algorithm of choice. Source nodes transmit $R R E Q$ and $D A T A$ messages to the sink node using the proposed Iso Persistent Field Routing.

\section{EXPERIMENTAL EVALUATION}

The experiments were performed using the SIDnet-SWANS [1], [7] simulator, which is an adapted version of JiST-SWANS [2] for WSN applications. We simulated 750 homogeneous nodes having the following configuration: (i) $20 \mathrm{kbps}$ radio data rate on the MAC802.15.4 protocol, (ii) 5 seconds idle-tosleep interval (i.e., nodes not along an active route go to sleep after 5 seconds of idle time, to preserve battery power), and (iii) power consumption characteristics based on the Mica2 Motes specs. To reduce the simulation time, while preserving the validity of the observations, a small fully-charged battery with an initial capacity of $35 \mathrm{mAh}$ powered each node, for a projected lifespan of several tens of hours under moderate load. The evaluations focus on long-term continuous queries (transferring of large amounts of data). 


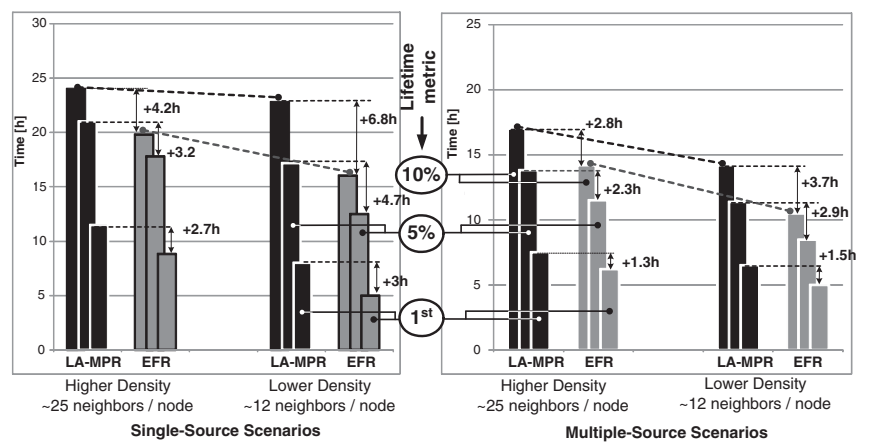

Fig. 5. Impact of network density on lifetime. For a density of 12 neighbors per node, LA-MPR performs significantly better than EFR. As the density increases, the difference in performance decreases.

We compare EFR [14] with our proposed LA-MPR approach in different scenarios. We configured 6 single-source and 3 multi-source scenarios, as illustrated in Figure 4. Each scenario was tested for 2 densities ( 25 an 12 neighbors per node, on average) and for 3 different number of paths between source(s) and sink (15, 30 and 50 paths). Each tuple [scenario (9), density (2), number of paths (3)] was tested on 30 different random and uniformly distributed deployments, resulting in a total of 1620 experiments.

It is important to remark that in our simulation setup, we vary the network density by adjusting the length of the sensing area rather than changing the power of the transceiver of individual nodes, in order to maintain consistency across simulations with respect to energy consumption.

Impact of Network Density on Network Lifetime. First let us expose EFR's main weakness: the number of paths is limited by the number of neighbors. This leads to energyimbalances, and as a consequence, shorter network lifetime.

Figure 5 depicts scenarios where the number of paths is 50 (greater than the number of neighbors in both densities). The bars represent the average over all single and multiple source deployments presented in Figure 4 (i.e. over 180 instances for single-source and 90 instances for multi-source).

Figure 5 gives a break-down for 3 different network lifetime metrics (10\% dead nodes, $5 \%$ dead nodes, first node dead). For a lifetime metric of $10 \%$ of dead nodes and a density of 12 nodes per neighbor, LA-MPR achieves 6.8 hours (40\%) of additional lifetime in single source scenarios, and 3.7 hours $(35 \%)$ in multiple source scenarios. When the lifetime metric is reduced to $5 \%$ of dead nodes or to the first dead node, the improvements are even higher for single source scenarios $(60 \%)$. This proves the effectiveness of field-persistent forwarding in unmerging paths in spite of the initial limitation imposed by the number of neighbors.

When the density of the network increases to 25 neighbors per node, the difference between EFR and LA-MPR decreases (between $15 \%$ and $30 \%$ for single source, and $20 \%$ for multiple sources). This occurs due to the fact that path-merging effects decrease as the density increases.

Figure 5 also shows that the lifetime of both routing

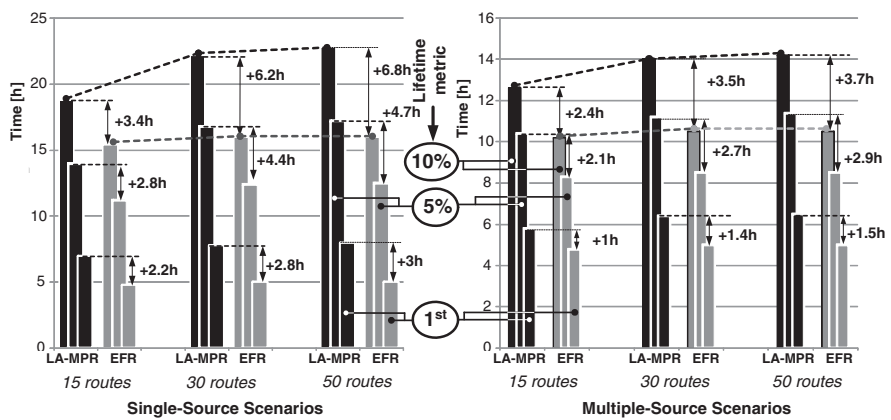

Fig. 6. Impact of path diversity on lifetime. For a density of 12 neighbors per node, increasing the number of paths improves the performance of LAMPR due to its "unmerging" characteristic, while EFR does not benefit from adding more paths.

schemes degrade with reduced network density. This happens because in sparser networks the path-length increases and consequently more energy is consumed on each end-to-end transmission (recall that the density is modified by extending the area instead of decreasing the transmission range).

Impact of Path Diversity on Network Lifetime. Now we present the impact of path diversity on the lifetime of the network. Figure 6 gives a break-down of network lifetime metrics under 15, 30 and 50 routes in single-source and multi-source settings for a density of 12 neighbors per node.

One of the key motivations of multi-path routing is that a larger number of allowable routing paths should yield better energy load-balancing, and consequently, better lifetimes. However, Figure 6 shows that increasing the number of paths has negligible effects on EFR because in all cases the number of paths is greater than the number of neighbors. On the other hand, LA-MPR shows an improvement when the number of paths is increased from 15 to 30 , but not a significant improvement from 30 to 50. This happens because there exists a maximum number of admissible paths, a saturation point, beyond which no additional lifetime gain can be obtained.

The important lesson of this subsection is that the saturation point of path diversity represents an important configuration parameter for field-based routing schemes, and hence should not be considered lightly.The advantage of LA-MPR is that it increases significantly the path-saturation point and hence allows for better load balancing.

Work-load Balancing. We compare load balancing by evaluating the standard deviation of the residual energy of the relay nodes that are involved in routing. Figure 7 shows that for a network density of 12, LA-MPR achieves significant improvements over EFR in both single-source (68\%) and multi-source $(56 \%)$ scenarios. For a network density of 25 , this difference decreases, confirming the key proposition of this paper: Due to its "unmerging" characteristic, LA-MPR efficiency increases as the network density decreases. Towards the end of simulation, the overall standard deviation decreases because most nodes' reserves converge towards the residual energy limit (5\%). 

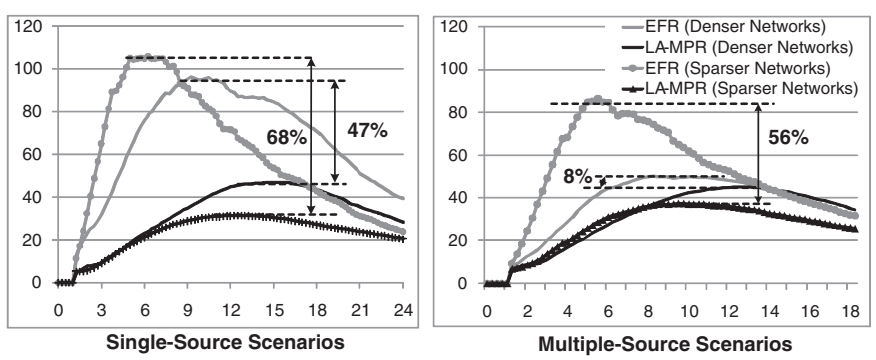

Fig. 7. Workload-balancing performance (standard deviation). Given that LA-MPR utilizes a larger fraction of the network to share the communication costs, the energy balance is significantly better in sparser networks.

\section{RELATED WORK}

Multipath routing has been identified as an alternative for load balancing, but not just any multipath technique would do. In [6], the authors show that in order to be effective, multipath routing should not select the $K$-shortest paths but rather select paths that spread the traffic across the network. Based on this insight, several important contributions have been proposed for single-sink single-source scenarios [3], [12], [15], [18]. However, these techniques have a major limitation on singlesink multiple-source scenarios: the various simultaneous paths between sources and sink would intersect each other creating severe contention problems at the MAC layer.

In order to avoid MAC collisions of multi-path routing in single-sink multiple-source scenarios, the community have used electric-field routing. The wide coverage of the electric fields allows the spreading of paths across the network, and the attraction-repulsion characteristic of the electric charges determines mutually exclusive routing areas for each source, limiting MAC collisions.

In [14], Nguyen et al. describe a distributed, stateless, multi-path electrostatic routing scheme (EFR). Their approach demonstrates scalability, robustness, higher delivery ratio and lower overhead compared to LAR, DREAM, GPSR and AOMDV. However, EFR is oblivious to the problem of network boundaries and does not perform well in sparse networks. Kalantari et al. [8] proposes a centralized solution for the boundary problem. The solution requires a-priori information about traffic demands and node positions. On a similar line of work, Toumpis and Tassiulas [17] shows that an optimal placement of nodes between a set of sources and sinks can be used to solve boundary problems.

Field-based routing has also been used to optimize other metrics (besides energy balancing), such as routing in networks with mobile sinks [16], service discovery in MANETs [10] and congestion avoidance over the Internet [4].

The key contributions of our work with respect to the state of the art are: (i) Field-Persistent Routing and (ii) method of images for boundary conditions. These mechanisms permit a better load balancing of the network.

\section{CONCLUSIONS}

In this work we addressed the problem of prolonging the lifetime of Wireless Sensor Networks (WSN) by enhanc- ing the performance of electrostatic-field routing. The main contribution of our work has been two-fold: (i) identify the shortcomings of EFR as network density decreases and (ii) propose 2 novel techniques to overcome these limitations.

In general, in multi-path routing, the more paths, the better the load balancing. However, in EFR as the network density decreases, the number of paths between source and sink is limited by the degree of the source (number of neighbors). In order to solve these problems we proposed Field-Persistent Routing and Method of Images. Field-Persistent routing removes to a large extent the limitation imposed by the number of neighbors, and the method of images avoids path merging in the borders of the network. Our results show that these techniques reduce the energy variance of sparser networks between $50 \%$ and $70 \%$, and increase the network lifetime between $30 \%$ and $40 \%$.

\section{REFERENCES}

[1] http://www.ece.northwestern.edu/ peters/sensors/.

[2] http://jist.ece.cornell.edu/index.html.

[3] S.J. Baek and G. de Veciana. Spatial energy balancing through proactive multipath routing in wireless multihop networks. IEEE/ACM TON '07.

[4] A. Basu, A. Lin, and S. Ramanathan. Routing using potentials: a dynamic traffic-aware routing algorithm. In SIGCOMM '03.

[5] J. Deogun, S. Das, H. Hamza, and S. Goddard. An Algorithm for Boundary Discovery in Wireless Sensor Networks, pages 343-352. 2005.

[6] Y. Ganjali and A. Keshavarzian. Load balancing in ad hoc networks: Single-path routing vs. multi-path routing. In INFOCOM ' 04 .

[7] O. Ghica, G. Trajcevski, P. Scheuermann, Z. Bischoff, and N. Valtchanov. Sidnet-swans: A simulator and integrated development platform for sensor networks applications. SenSys, 2008.

[8] M. Kalantari and M. Shayman. Routing in wireless ad hoc networks by analogy to electrostatic theory. In ICC '04.

[9] S. Kwon and NB. Shroff. Paradox of Shortest Path Routing for Large Multi-Hop Wireless Networks. In INFOCOM '07.

[10] V. Lenders, M. May, and B. Plattner. Service discovery in mobile ad hoc networks: A field theoretic approach. Pervasive and Mobile Computing, 1(3):343-370, 2005.

[11] W. Luo, W. Liu, and Y. Zhang. Performance optimizatin using multipath routing in mobile ad hoc and wireless sensor networks. Combinatorial Optimization in Communication Networks, 2, 2005.

[12] A. Mei and J. Stefa. Routing in outer space: fair traffic load in multi-hop wireless networks. In MobiHoc '08.

[13] R. Nagpal, H. Shrobe, and J. Bachrach. Organizing a global coordinate system from local information on an ad hoc sensor network. In IPSN 03 .

[14] N. T. Nguyen, A. Wang, P. Reiher, and G. Kuenning. Electric-field-based routing: a reliable framework for routing in MANETs. SIGMOBILE Mob. Comput. Commun. Rev., 8(2):35-49, 2004.

[15] L. Popa, A. Rostamizadeh, R. Karp, C. Papadimitriou, and I. Stoica. Balancing traffic load in wireless networks with curveball routing. In MobiHoc '07.

[16] J. Tateson and I.W. Marshall. A Novel Mechanism for Routing in Highly Mobile Ad Hoc Sensor Networks. LNCS, pages 204-217, 2004.

[17] S. Toumpis and L. Tassiulas. Packetostatics: deployment of massively dense sensor networks as an electrostatics problem. In INFOCOM 2005.

[18] G. Trajcevski, O. Ghica, and P. Scheuermann. CAR: controlled adjustment of routes and sensor networks lifetime. In $M D M$ ' 06 .

[19] G. Trajcevski, O. Ghica, R. Shubotz, M. Zuniga, P. Scheuermann, and M. Hauswirth. Discrepancy and multipath routing in wireless sensor networks. NWU-EECS-09-18.

[20] S. Wu and K. Selçuk Candan. Power-aware single- and multipath geographic routing in sensor networks. Ad Hoc Networks, 5(7), 2007. 